\title{
Gravitational field and mass
}

Yin Zhu

Agriculture and Rural Department of Hubei Province, Wuhan, China

Email: waterzhu@163.com

(September 1, 2021; November 26, 2021, v2)

\begin{abstract}
It is extremely fascinating and astonishing that the gravitational field on the surface of a neutron star is with a relativistic mass density of $2.65 \times 10^{16} \sim 5.87 \times 10^{18} \mathrm{kgm}^{-3}$ which can be larger than the mass density of the neutron stars $\left(\sim 10^{17} \mathrm{kgm}^{-3}\right)$. Therefore, it is the author's first intuitional imagining that this field could directly convert into mass. In so strong a gravitational field, electron and proton could be produced directly from graviton-photon collision. The gravitational field exists in everywhere in our universe. No vacuum that the region of a space is "empty" does exist. A particle is clearly always being acted on by the gravitational field. The quantum vacuum fluctuation and vacuum polarization need be re-understood with the interaction between photon and gravitational field. Therefore, the gravitational field is naturally one of the foundations of modern physics.
\end{abstract}

Key words: Super strong gravitational field; Mass-Gravitational field conversion; Graviton-photon conversion; Graviton-photon to electron-proton conversion; Vacuum fluctuation; Vacuum polarization 
Before 1900, the gravitational field is not needed for the foundation of physics. Therefore, it was omitted traditionally although it has to be considered in modern physics. In 1643, as Torricelli[1] presented the concept of vacuum, the gravitational field had not been known. So, in the Torricellian vacuum, the gravitational field is not excluded. It is a fact that the Torricellian vacuum is useful in many areas of current physics. For example, in the thermodynamics and statistic mechanics, based on the Torricellian vacuum, the motion of particles can be well described. But, now, the super strong gravitational field was observed. The gravitational acceleration on the surface of a neutron star is $2 \times$ $10^{11} \sim 3 \times 10^{12}$ times that of the Earth.[2]

It is important, a gravitational field is with energy. It is well-known that there is $m g h=\frac{1}{2} m v^{2}$. The gravitational field can make a mass $m$ moved with the energy of $\mathrm{E}=\frac{1}{2} m v^{2}$ as the mass is freely falling in the field. The energy of the field of the Earth can be accurately measured. And, it is known that the energy density of a gravitational field, $\quad \mathrm{U}_{g}=\frac{g^{2}}{8 \pi G},[3]$ is determined with the gravitational acceleration $g$ and the Newtonian universe gravitational constant G.

From $\mathrm{E}=\mathrm{m} c^{2}$ we know, correspondent to $\mathrm{U}_{g}=\frac{g^{2}}{8 \pi G}$, there is a relativistic mass density $d_{g}=\frac{1}{c^{2}} \frac{g^{2}}{8 \pi G}$. It means that, in the region of a space in anywhere that we now call it the vacuum there is an energy density of $\mathrm{U}_{g}=$ $\frac{g^{2}}{8 \pi G}$ and a relativistic mass density of $d_{g}=\frac{1}{c^{2}} \frac{g^{2}}{8 \pi G}$ of the gravitational field.

On the surface of the Earth, $g_{e}=10 \mathrm{~ms}^{-2}$, correspondently, $U_{g e}=\frac{g^{2}}{8 \pi G} \approx 5.97 \times 10^{10} \mathrm{kgm}^{-1} s^{-2}$. In this case, $d_{g e}=\frac{1}{c^{2}} \frac{g^{2}}{8 \pi G} \approx 6.63 \times 10^{-7} \mathrm{kgm}^{-3}$ is very little. So, it can be neglected in the traditional physics. For example, the mass density of the hydrogen gas is $d_{H}=8.9887 .5 \times 10^{-2} \mathrm{kgm}^{-3}$. Therefore, the gravitational field on the surface of the Earth with the relativistic mass density of $d_{g e}=\frac{1}{c^{2}} \frac{g^{2}}{8 \pi G} \approx 6.63 \times 10^{-7} \mathrm{kgm}^{-3}$ has little effect on the mass density of the hydrogen gas. But, for a super strong gravitational field (Here, we call the gravitational field with $d_{g}=\frac{1}{c^{2}} \frac{g^{2}}{8 \pi G} \geq 1 \times 10^{3} \mathrm{kgm}^{-3}$ the super strong field), the energy density and relativistic mass density are very big. For example, the gravitational acceleration on the surface of a neutron star is $g_{n}=2 \times$ $10^{11} \sim 3 \times 10^{12} g_{e}$. Correspondently, there are $\mathrm{U}_{g n}=\frac{g^{2}}{8 \pi G} \approx 2.39 \times 10^{33} \sim 5.37 \times 10^{35} \mathrm{kgm}^{-1} \mathrm{~s}^{-2}$ and $d_{g n}=\frac{1}{c^{2}} \frac{g^{2}}{8 \pi G} \approx 2.65 \times 10^{16} \sim 5.90 \times 10^{18} \mathrm{kgm}^{-3}$. We know, the density of water is $10^{3} \mathrm{kgm}^{-3}$; the largest mass density in the Earth is $2.259 \times 10^{4} \mathrm{~kg} / \mathrm{m}^{3}$ (the osmium). The relativistic mass density of the gravitational 
field on the surface of a neutron star is much larger than the largest mass density in the Earth. In any case, such strong a field cannot be omitted.

The mass density of a neutron star is $d_{n} \approx \sim 10^{17} \mathrm{kgm}^{-3}$. Therefore, the relativistic mass density of the gravitational field of a neutron star can be larger than the mass density of the neutron stars.

For a star and its field, from $d_{g}=\frac{1}{c^{2}} \frac{g^{2}}{8 \pi G}$ and $d_{m}=M /\left(\frac{4}{3} \pi r^{3}\right)$, for $d_{g} \geq d_{m}$, there is

$$
G \frac{M}{r} \geq 6 c^{2}
$$

or

$$
\mathrm{r} \leq \frac{1}{6} \frac{G M}{c^{2}}
$$

$\frac{2 G M}{c^{2}}$ is the Schwarzschild radius. It indicates that, as the radius of a celestial body is less than $\frac{1}{12}$ times the Schwarzschild radius, the relativistic mass density of the gravitational field on the surface of the star is larger than the mass density of the star.

It is extremely fascinating and astonishing that the relativistic mass density of the gravitational field is larger than the mass density of the star. The first intuitional imagining is that the mass, including electron and atomic nucleus, could be directly converted from the field. In theory, it was presented that, graviton could be converted into photon, and vice versa.[4,5] This conversion was widely studied in the condition of strong field, such as that of blackhole.[611] The electron-positron pair and hadronic pair produced from high energy photon-photon collision was experimentally observed.[12-20] The photons can annihilate into: 1) a charged and neutral pair such as $\gamma \gamma \rightarrow$ $\left.\mathrm{W}^{+} W^{-}, \mathrm{q} \overline{\mathrm{q}}, \gamma \gamma \rightarrow Z^{0} Z^{0}, 2\right)$ lepton pairs, and 3) charged and neutral Higgs. Therefore, according to current theory, proton could be produced from the sub-collision of graviton-photon conversion: high energy photon produced from graviton-photon collision-leptons produced from photon-photon collision - proton produced from the collision of these particles-Higgs mechanics makes virtual particles transferred into real ones. But, here, it is conjectured that the proton could be produced directly from the high energy graviton-photon collision.

A gravitational field is emitted from a mass. A ray of gravitational field can be shown as Figure 1 which is with a direction along the radial direction of the gravitational field. The graviton is with a spin \pm 2 . The direction and spin 
\pm 2 could indicate that, the ray of gravitational field is chiral. If electron and proton could be produced from photongraviton scattering, the chirality of the field should result in that only one kind of electron and proton can be produced, instead of that the matter-antimatter pair is produced. This may be the reason that the antimatter is very rare.

Figure 1 is very familiar with many people. But, it has not been taken as an evidence for that a gravitational field is chiral. It is noted that the photon is with spin \pm 1 . A light ray also is with a direction. Therefore, the photon-photon collision could be chiral. But, now, it is believed that only the matter-antimatter pair can be produced from the $\gamma \gamma$ collision. Therefore, it is questioned that whether or not the production from the $\gamma \gamma$ collision can be chiral.

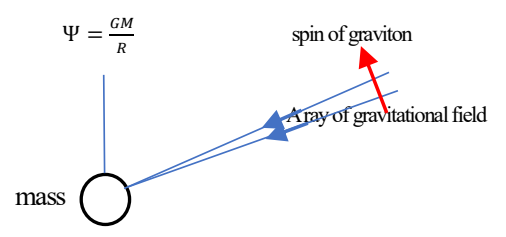

Figure 1. The chirality of a gravitational field. 1) At any a point of a gravitational field, there is a gravitational potential $\Psi=\frac{G M}{R}$. 2) The gravitational field is with a gravitational acceleration $g=\frac{G M}{R^{2}}$ which is directed toward to the central of the mass. 3) The graviton is with the spin \pm 2 .

In the Breit-Wheeler process,[21] the condition for the electron-positron pair produced from high energy photonphoton collision is $E_{\gamma 1} E_{\gamma 2}(1-\cos \varphi) \geq 2 m_{e}^{2} c^{4}$, where $E_{\gamma 1}$ and $E_{\gamma 2}$ are the energy of the photons, $m_{e}$ is the mass of the electron. Analogy to the condition, as the energy of the graviton $E_{g}$ is large enough, the electron and proton could be produced from photon-graviton collision. Therefore, for the mass of electron $m_{i}$ and that of proton $m_{j}$, under the condition of

$$
E_{\gamma} E_{g}(1-\cos \varphi) \geq 2 m_{i} m_{j} c^{4}
$$

the electron and proton could be directly produced from graviton-photon collision. 
From the conservation of charge and spin, the Feynman diagram for the photon-graviton collision could be expressed as Figure 2. It means that, before collision, the sum of the charge for graviton and photon is $\mathrm{q}=0 \mathrm{e}+$ $0 \mathrm{e}=0$ and the sum of the spin for the graviton and photon is $\mathrm{s}=-2+1=-1$ or $\mathrm{s}=2-1=+1$; while after collision, the sum of the charge is $\mathrm{q}=-1 \mathrm{e}+1 \mathrm{e}=0$ and the sum of spin is $\mathrm{s}=-\frac{1}{2}+\left(-\frac{1}{2}\right)=-1$ or $\mathrm{s}=\frac{1}{2}+\frac{1}{2}=1$. However, now, Figure 2 is only an imagined one. But, it could be checked observationally.

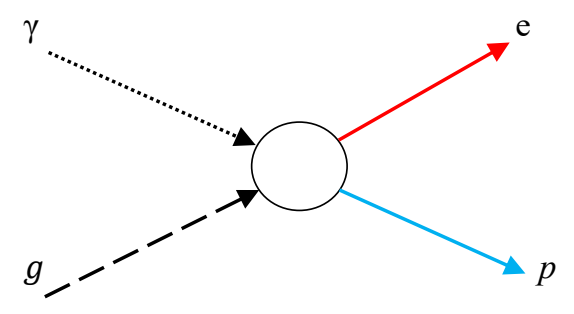

Figure 2. Electron-proton produced from photon-graviton collision: $\gamma \boldsymbol{g} \rightarrow \boldsymbol{e p}$. Because of the direction of the gravitational field and the spin of graviton, it is conjectured, the proton (p) and electron (e) could be directly produced from the graviton $(g)$-photon $(\gamma)$ collision.

The mass of a proton is much larger than that of an electron. In $E_{\gamma} E_{g}(1-\cos \varphi) \geq 2 m_{i} m_{j} c^{4}$, a critical energy for the graviton is needed to produce a proton. According to $\mathrm{U}_{g}=\frac{g^{2}}{8 \pi G}$, the critical energy is determined with the distance $\mathrm{R}$ from the source mass as shown in Figure 3. Here, we call $\mathrm{R}$ the critical distance. It determines that, inside the critical distance, the proton could be produced. While out of this distance, only little proton could be produced by the Heisenberg's principle of uncertainty. Therefore, the numbers of the protons out of the critical distance is sharply reduced to almost zero as shown in the Figure 3.

The observation of Sgr A* showed that there are the ionized and neutral gas, dust and hard x-ray which form the clumpy streamers or filaments orbiting around Sgr A*.[22] And, the reason for the origin of these matters are being discussed. If electron, proton and other particles could be produced from photon-graviton collision, this observation 
could be understood and explained. And, the critical distance could be observed from Sgr A*. It is important, the Sgr A* is a good object for observing the super strong gravitational field.

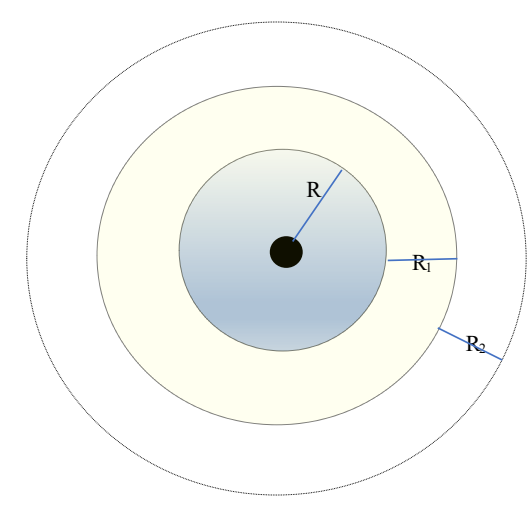

Figure 3. The distribution of the protons and other particles around a mass with super strong field. Determined with $E_{\gamma} E_{g}(1-\cos \varphi) \geq 2 m_{i} m_{j} c^{4}$, the distribution of the particles produced from gravitonphoton collision should be layered sharply. The protons should be assembled inside the R region. Out of R, the protons are almost zero. In the $\mathrm{R}_{1}$ region, there should be the leptons and the hadrons with the mass less than the proton. In the $\mathrm{R}_{2}$ region, the leptons are very little.

The quantum fluctuation was observed experimentally.[23,24] Now, the quantum fluctuation is also called "vacuum fluctuation". Quantum vacuum fluctuation is a fundamental concept in the quantum field theory.[25-28] It is thought that a real photon could be generated from a vacuum. In current quantum field theory, a quantum electrodynamics vacuum is the lowest energy state of the electromagnetic field when the fields are quantized. Therefore, it is thought that the deep space is approximations of the vacuum with a few hydrogen atoms, a lots of photons, the cosmic background radiation and neutrinos. And, the artificial vacuum can be arrived at. It seems that, the difference between the quantum electrodynamics vacuum and the Torricellian vacuum is little. In both of them, the gravitational field has not been excluded.

It is clear and certain, in the experiments, $[23,24]$ the electrons interacted by the gravitational field cannot be omitted. The photon-graviton collision has to be considered. Therefore, from Refs. [4,5], I prefer to believe that the observed 
quantum fluctuation[23,24] is an effect of graviton-photon collision and the conversion of graviton into photon. In this sense, it could be concluded that the graviton with the graviton-photon interaction was detected in quantum fluctuation.

It is clear, the quantum fluctuation is related with Heisenberg's principle of uncertainty. Therefore, as the vacuum zero-point energy is replaced by the energy of the gravitational field, the current explanation about the quantum fluctuation only need be improved.

The vacuum polarization also need be re-understood with the gravitational field. It is thought that the "vacuum polarization" was observed in lab[29] and from the strong magnetic field of the neutron star.[30,31] On a neutron star, the effect of gravitational field is super strong. It is clear, it is not suitable to omit the effect of such strong a field on the electromagnetic field or photon.

Light bending by gravity and gravitational redshift were well known nowadays. Under the condition of the super strong gravitational field, some of strong and new effects could be possibly discovered by analogy to that in the electrodynamics. For example, analogy to the Zeeman effect, as a light ray running through a strong gravitational field, the direction of this light ray could be changed by the gravitational field and this change could be very large. If there are the gas around a star, the effect analogous to the Kerr effect and Faraday effect should be produced. Therefore, the observation in Ref.[30,31] may be a new kind of the quantum effect of the super strong gravitational field on electromagnetic field and photon.

It was presented that the photon and electron may be originated from vacuum under the condition of chiral symmetry.[32] But, if the gravitational field is considered, it could be concluded that the photon and electron can be converted from the gravitational field and this conversion is chiral.

Historically, the first studied field is the gravitational field. In 1600s, René Descartes presented that the attractive force of the gravity is produced from the ether vortex.[33] But, in current physics, the theory of gravitational field is only based on the analogy to that of the electromagnetic field. It is believed that the gravitational field of a mass is analogous to the electric field of an electron. But, different from the interacting electric field which can be neutralized for that there are positive and negative charges, the interacting gravitational field is invariant for that gravitational 
field cannot be distinguished as negative or positive ones. It is clearly shown that this analogy is invalid. In strict scientific standard, a physics theory only can be developed from observation and experiment. Therefore, it can be thought that, now, we have not had a complete theory for the gravitational field.

Factually, observation and experiment have laid the foundation to develop a scientific theory of gravitational field. First, Newtonian theory of gravity with the observation of celestial orbit and with the experiment of artificial orbit laid the foundation to develop the theory of the interaction of gravitational field. Newton demonstrated that the repulsive gravity can be produced from the interaction of gravitational field.[34,35] (But, Newton's repulsive gravity has been omitted till now.) From Newton's repulsive gravity, it could be concluded that the interacting gravitational field could be limited.[36] Second, the gravitational redshift and the light bending by gravity were observed. It showed that the energy of photon and the motion of a light ray can be varied by gravity. From the gravitational redshift, according to the law of energy conservation, it could be concluded that, the energy of the graviton can be correspondently varied by the photon.[37,38] Therefore, the gravitational acceleration of the Earth could be varied with strong electric and magnetic field. Third, the quantum fluctuation[23, 24] and "vacuum" polarization[29-31] were observed. As the gravitational field is considered, the quantum fluctuation could be understood as that the graviton is converted into the photon[4,5] and the "vacuum" polarization could be understood as that the motion of light ray and the energy of photon are changed by the gravitational field.

Therefore, it is possible that the graviton with the graviton-photon interaction was detected in the experiments $[23,24]$ for quantum fluctuation and could be detected in the gravitational-magnetic/electric field interaction[37,38].

These features for a gravitational field were well observed: 1) It is measurable. From mgh $=\frac{1}{2} m v^{2}$, the energy of a gravitational field can be accurately measured. 2) A gravitational field is emitted from a body. So, a gravitational field is with a direction. 3) The gravitational field is not uniform in the universe. There are the super strong gravitational field and very weak ones. Therefore, the gravitational field is not a kind of ether. Also, it is not dark energy or dark matter.

The first intuitional imagining from the super strong gravitational field is that the field could be directly converted into mass for that the relativistic mass density of the field is much larger than the largest mass density in the Earth. The conversion between the graviton and photon was theoretically studied and the electron-positron pair and 
hadronic pair produced from high photon-photon collision were observed. And, the charged and neutral Higgs produced from photon-photon collision was studied[12-20] Therefore, in current theory, mass could be produced from photon-graviton collision. In this work, it is presented that the electron and proton could be directly produced from photon-graviton collision.

Now, the super strong gravitational field has been observed and the conversion between photon and graviton may have been measured[23,24] and was studied under the condition of strong field.[6-11] Therefore, it is the time to observe the graviton to other particles conversion in the super strong filed.

It is well known that a mass is always with a gravitational field. If the electron and proton could be produced from the photon-graviton collision, it should mean that, the mass and gravitational field could be converted into each other. But, it has not been known that which of them is the premise. And, it is very mysterious that what is the reason that can make an object with a relativistic mass density of $d_{g n}=\frac{1}{c^{2}} \frac{g^{2}}{8 \pi G} \approx 2.65 \times 10^{16} \sim 5.90 \times 10^{18} \mathrm{kgm}^{-3}$ of a field and that why it has not become mass.

And, the relativistic mass density of the magnetic field also is very large. The magnetic field on a magnetic star can be $\mathrm{B} \approx 1 \times 10^{9} \mathrm{~T} \cdot[30,31]$ The relativistic mass density of this magnetic field is $d_{m}=\frac{1}{c^{2}} \frac{B^{2}}{2 \mu_{0}} \approx$ $4.40 \times 10^{6} \mathrm{kgm}^{-3}$. It is $10^{3}$ times the density of the water. Therefore, the large relativistic mass density of the field is the common nature for both gravitational and magnetic fields.

New discovery is always remodeling the foundation of physics. The super strong gravitational field has been observed for several decades. It need be integrated into the foundation of modern physics. The gravitational field with the relativistic mass density of $10^{12} \sim 10^{14}$ times the largest mass density in the Earth clearly shows that the current concept of vacuum, including both Torricellian vacuum and quantum electrodynamics vacuum, need be changed. The quantum electrodynamics vacuum is one of the foundations the quantum field theory. Therefore, the results based on the foundation need be changed. It seems that, as the concept and the term of vacuum are replaced by that of the gravitational field, this change should not affect the main conclusions, including the experimental results, based on the concept and term of vacuum.

It was believed that ether, vacuum and cosmic background radiations (CMBR) are the objects that exist in 
everywhere of our universe. The ether was taken as the main foundation of physics in 1900s.[39] Now, the vacuum is taken as the foundation of several areas of physics. But, the ether has not been observed. And, in theory, there is the vacuum while in practice vacuum neither does exist naturally nor can be made artificially. And, the CMBR is very weak, it only can be observed in a limit distance. Therefore, gravitational field is the only object that exists in the observable space of our universe. In the experiments and observations about the quantum fluctuation[23,24] and "vacuum" polarization in lab[29] and in the super strong gravitational field,[30,31] the effect of gravitational field on the law of physics has to be considered. Thus, the gravitational field naturally is one of the foundations of physics.

\section{References}

[1] Jervis-Smith and Frederick John, Evangelista Torricelli. Oxford University Press (1908)

[2] Bejger M. \& Haensel P., Surface gravity of neutron stars and strange stars, Astron. Astrophys. 420, 987 (2004)

Gendy M. A. \& Morsink S. M., Universality of the Acceleration due to Gravity on the Surface of a Rapidly Rotating Neutron Star, The Astrophys. J., 791, 78 (2014)

[3] Lyinden-Bell D. \& Kartz J., Gravitational field energy density for spheres and black holes, Mon. Not. Astro. Soc., $213,(1985)$

Miller J. S., On the Energy Density in a Gravitational Field, The American Journal of Physics, 18 (4) (1950)

[4] De Logi W. K. \& Mickelson A. R., Electrogravitational conversion cross-sections in static electromagnetic fields, Phys. Rev. D 16, 2915-2927 (1977)

[5] Bjerrum-Bohr N. E. J., Holstein B. R., Plant'e L., and Vanhove P., Graviton-photon scattering, Phys. Rev. D 91, 064008 (2015), arXiv:1410.4148 [gr-qc]

[6] Olson D. W. and Unruh W. G., Conversion of electromagnetic to gravitational radiation by scattering from a charged black hole, Phys. Rev. Lett. 33, 1116-1119 (1974).

[7] Bartolo N., Hoseinpour A., Orlando G., Matarrese S., Zarei M., Photon-graviton scattering: A new way to detect anisotropic gravitational waves? Phys. Rev. D 98, 023518 (2018)

[8] Chandrasekhar S., On the equations governing the perturbations of the Reissner-Nordstr" om black hole, Proc. Roy. Soc. Lond. A 365, 453-465 (1979).

[9] Gunter D. L., A study of the coupled gravitational and electromagnetic perturbations to the reissner nordstr" om black hole: The scattering matrix, energy conversion, and quasi-normal modes, Philosophical Transactions of the Royal Society of London. Series A, Mathematical and Physical Sciences 296, 497-526 (1980). 
[10] Breuer R. A., Rosenbaum M., Ryan M. P. and Matzner R. A., Gravitational electromagnetic conversion scattering on fixed charges in the Born approximation, Phys. Rev. D 23, 305-311 (1981).

[11] Hadj M. and Dolan S. R, Conversion of electromagnetic and gravitational waves by a charged black hole, arXiv:2106.09731v2 (2021).

[12] Brodsky, S. J., Photon--Photon Collisions --- Past and Future, Acta Physica Polonica B, 37, 619 (2006)

[13] Urner D., Review of Two-Photon Interactions, arxiv: hep-ex/0309045

[14] Balakin V. E., Budker G. I., Pakhtusova E. V., Sidorov V. A., et al, Experiment on $2 \gamma$-quantum annihilation on the VEPP-2, Phys. Lett. B 34, 99 (1971).

[15] Baldini Celio R., Capon G., Del Fabbro R., De Santis P., et al., Experimental results on photon-photon interactions at adone, Phys. Lett. B 86, 239 (1979)

[16] Burke D. L. et al., Positron Production in Multiphoton Light-by-Light Scattering, Phys. Rev. Lett. 79, 1626 (1997)

[17] Adam, J., et al., Measurement of e + e Momentum and Angular Distributions from Linearly Polarized Photon Collisions, Phys. Rev. Lett. 127, 5 (2021)

[18] Demirci M., Precise predictions for charged Higgs boson pair production in photon-photon collisions, Nuclear Physics B, 961, 115235 (2020)

[19] The DELPHI Collaboration, Study of tau-pair production in photon-photon collisions at LEP and limits on the anomalous electromagnetic moments of the tau lepton, The European Physical J. C, 35, 159-170 (2004)

[20] Diehl M., Kroll P. \& Vogt C., Two-photon annihilation into baryon-antibaryon pairs, The European Physical Journal C 26, 567-577 (2003)

[21] Breit G. and Wheeler J. A., Collision of two light quanta, Phys. Rev. 46, 1087 (1934)

[22] Genzel R., Eisenhauer F. and Gillessen S., The Galactic Center Massive Black Hole and Nuclear Star Cluster, Reviews of Modern Physics, 82.4, 3121-3195 (2010)

[23] Riek C., Seletskiy V. D., Moskalenko S. A., Schmidt J. F., et al., Direct sampling of electric-field vacuum fluctuations, Science, 320, 420, (2015)

[24] Halbhuber M., Mornhinweg J., Zeller V., Ciuti C., Bougeard D., et al, Non-adiabatic stripping of a cavity field from deep-strongly coupled electrons, Nature Photonics (2020)

[25] Dyson F., The radiation theories of Tomonaga, Schwinger and Feynman, Phys. Rev. 75, 486, (1949)

[26] Dirac P., Proc. Roy. Soc. A 209, 291 (1951)

[27] Feynman R., Nobel lecture, reproduced in Science 153, 699 (1966) 
[28] Günter Scharf, Finite Quantum Electrodynamics - The Causal Approach, Berlin: Springer-Verlag, 1995, 2nd edition

[29] Levine I., TOPAZ Collaboration, Measurement of the Electromagnetic Coupling at Large Momentum Transfer, Physical Review Letters. 78: 424 427 (1997)

[30] Lai D., Ho W. Polarized X-Ray Emission from Magnetized Neutron Stars: Signature of Strong-Field Vacuum Polarization, Physical Review Letters, 91(7):071101 (2003)

[31] Mignani R. P., Testa V., Gonzalez Caniulef D., Taverna R., et al, Evidence for vacuum birefringence from the first optical-polarimetry measurement of the isolated neutron star RX J1856.5-3754, MNRAS, 465, 492-500 (2017)

[32] Levin M. and Wen X. G., Quantum ether: photons and electrons from a rotor model, Physical Review B, 73.3 (2006)

[33] Gaukroger S., Rene Descartes: An Intellectual Biography, Clarendon Press (1997)

[34] Newton I., Philosophiae Naturalis Principia Mathematica, (Harvard University, Cambridge, MA, 1972)

[35] Gutzwiller M. C., Moon-Earth-Sun: The oldest three-body problem, Review of Modern Physics, 70(2), 589$639(1998)$

[36] Zhu Y., Updating the Historical Perspective of the Interaction of Gravitational Field and Orbit in Sun-PlanetMoon System, International Journal of Astronomy and Astrophysics, 11, 343 (2021)

[37] Zhu Y., Gravitational-magnetic-electric field interaction, Results in Physics, 10, 794 (2018)

[38] Zhu Y., Gravitational-Magnetic-Electric Interaction in Controlling Relative Permittivity and Permeability[v2] $\underline{\text { Preprints }}$

[39] Rosser W.G.V., An Introduction to the Theory of Relativity (Butterworths, London, 1971)

Conflicts of Interest: The author declares no conflicts of interest regarding the publication of this paper. 